\title{
RELATIVISTIC REDUCTION OF ASTROMETRIC OBSERVATIONS
}

\author{
V. A. BRUMBERG \\ Institute of Theoretical Astronomy, 191187 Leningrad, U.S.S.R.
}

\section{Abstract}

The nonuniqueness of the quasi-Galilean coordinates of general relativity leads to the emergence of unmeasurable coordinate-dependent quantities in astronomical practice. One may offer three possible ways to overcome the related difficulties:

1. developing theoretical conclusions only in terms of measurable quantities

2. using arbitrary coordinates and developing an unambiguous procedure for comparing measurable and calculated quantities

3. agreement to utilize one and only one coordinate system.

In this paper we prefer the second way. After formulating the heliocentric planetary and geocentric satellite equations of motion, the general technique for relativistic reduction in astrometry and geodynamics is developed. Specific algorithms for the reduction of absolute and relative measurements are derived for the one- and the twobody problem. For illustration, the relativistic reduction of stellar parallaxes, Doppler satellite observations, navigation measurements with the aid of satellites and radiointerferometric measurements are presented in detail.

\section{Introduction}

The general theory of relativity in its most simple applications (Schwarzschild solution, weak-field approximation) is no longer seen as a theory under verification, but must be considered as a necessary framework in the discussiion of high-precision observations (0.001" in angular distance, $1 \mathrm{~ns}$ in time, $10^{-14}$ in frequency) and for the construction of accurate dynamical ephemerides $\left(10^{-8}\right.$ to $10^{-9}$ with respect to the main Newtonian terms). In the immediate future, relativistic reduction of astrometric observations will have to become as common as all classical types of reduction are today. Many authors are now working on different forms of relativistic reduction. The aim of this paper is to present a version of such reductions together with some actual equations of relativistic celestial mechanics and geodynamics.

Relativistic reduction of observations should take into account the dependence of 
measurements of time, angular distances and light frequency on the velocity of the observer and the value of the gravitational potential at the point of observation. (The last of these are specific for general relativity while a first approximation of the first one has been considered even in Newtonian physics.)

The most peculiar feature of general relativity is the arbitrariness of the coordinates used for the description of the gravitational field and space-time events. In general relativity, one cannot introduce the global Galilean (inertial) coordinates. Instead, one may use the quasi-Galilean coordinates. The metric of the gravitational field $\left(\mathrm{g}_{\mu \nu}\right)$ described in these coordinates, given by

$$
d s^{2}=g_{\mu \nu} d x^{\mu} d x^{\nu} ; \mu, \nu=0,1,2,3
$$

will differ little from the Galilean one $\left(\eta_{\mu \nu}\right)$ and will coincide with the latter at infinitely large distances from the attracting masses, to wit,

$$
\begin{aligned}
& \mathrm{g}_{\mu \nu}=\eta_{\mu \nu}+h_{\mu \nu} \text {, wi th }\left|h_{\mu \nu}\right|<<1 \text {, and } h_{\mu \nu} \rightarrow 0 \text {, for } r \rightarrow \infty \text {, } \\
& \text { also } n_{o o}=1, n_{o i}=0, n_{i j}=-\delta_{i j} ; i, j=1,2,3 \text {. }
\end{aligned}
$$

Quasi-Galilean coordinates $x^{\mu}$ are not unique and may be subjected to any arbitrary nonlinear transformation which preserves the relations (2). In the new quasi-Galilean coordinates $\tilde{\mathbf{x}}^{\mu}$, the equations of motion of bodies and light propagation as well as the solutions of these equations will differ from their analogues expressed in terms of $x^{\mu}$. This leads to the problem of the correspondence between measurable quantities (time, angular distances, light frequency) and the coordinate dependent quantities involved in theoretical calculations.

Such nonuniqueness of quasi-Galilean coordinates results in the intrusion of coordinate-dependent quantities into astronomical practice which are unmeasurable in principal. For example, the well-known numerical theories of motion of the major planets (DE-200) and the Moon (LE-200) developed at JPL, involve harmonic coordinates. Along with this, there exist similar theories developed in standard coordinates (Oesterwinter and Cohen 1972; Krasinsky et al. 1981). The difficulties resulting from the fact that different quasi-Galilean coordinates may be used in the treatment of any one problem would, in principle, be overcome in any of three ways: 1). refusing to employ coordinates as well as coordinate-dependent quantities, and constructing theories in terms of measurable quantities only 2). using arbitrary coordinates and developing unambiguous procedures to compare measurable and calculated quantities 3). an agreeement binding on every investigator (settled by the IAU, for example) to use one and the same specific system of coordinates. The first alternative seems too restrictive for theoretical constructions, whereas the third one constrains the freedom of a researcher to choose the coordinates best suitable for his problem. The developments in this paper are based on the second alternative. 
All the following relations are derived only in the post-Newtonian approximation valid for timely modern requirements.

\section{The gravitational field and the equations of motion of planets and satellites.} metric:

The gravitational field of non-rotating point masses is described by the following

$$
\begin{aligned}
& \mathrm{ds}^{2}=\left\{1-2 \Sigma_{\mathrm{i}} \frac{\mathrm{m}_{\mathrm{i}}}{\rho_{\mathrm{i}}}+2(\beta-\alpha)\left(\Sigma_{\mathrm{i}} \frac{\mathrm{m}_{\mathrm{i}}}{\rho_{\mathrm{i}}}\right)^{2}+(4 \beta-2) \Sigma_{\mathrm{i}} \frac{\mathrm{m}_{\mathrm{i}}}{\rho_{\mathrm{i}} \mathrm{j} \neq \mathrm{i}} \frac{\mathrm{m}_{\mathrm{j}}}{\mathrm{r}} \mathrm{ij}+\right. \\
& +2 \alpha \sum_{i} \frac{m_{i}}{\rho_{i}^{3}} j \sum_{i} m_{j}\left(\frac{1}{r_{i j}}-\frac{1}{\rho_{j}}\right)\left(\underline{\rho}_{i} \quad \underline{r}_{i j}\right)-\frac{1}{c^{2}}(2 \gamma+1) \sum_{i} \frac{m_{i}}{\rho_{i}} \underline{\underline{r}}_{i}^{2}+ \\
& \left.+\frac{1}{c^{2}}(\nu-1) \Sigma_{i} \frac{m_{i}}{\rho_{i}}\left[\dot{\underline{r}}_{i}^{2}-\underline{\rho}_{i} \ddot{r}_{i}-\frac{1}{\rho_{i}^{2}}\left(\underline{\rho}_{i} \underline{\dot{r}}_{i}\right)^{2}\right]\right\} \mathrm{c}^{2} \mathrm{dt}^{2}+ \\
& +\frac{2}{\mathrm{c}} \sum_{\mathrm{i}} \frac{\mathrm{m}_{\mathrm{i}}}{\rho_{\mathrm{i}}}\left[\left(2 \gamma+2-\alpha-\frac{1}{2} v\right) \underline{\underline{s}}_{\mathrm{i}}+\left(\alpha+\frac{1}{2} \nu\right) \frac{1}{\rho_{\mathrm{i}}^{2}}\left(\underline{\rho}_{\mathrm{i}} \underline{\underline{r}}_{\mathrm{i}}\right) \underline{\rho}_{\mathrm{i}}\right] \mathrm{d} \underline{\mathbf{r}} \cdot \mathrm{cdt}- \\
& -\left[\begin{array}{ll}
1+2(\gamma-\alpha) & \Sigma_{i} \frac{m_{i}}{\rho_{i}}
\end{array}\right] d \underline{r}^{2}-2 \alpha \Sigma_{i} \frac{m_{i}}{\rho^{3}}\left(\underline{\rho}_{i} d \underline{r}\right)^{2}
\end{aligned}
$$

Here, the main PPN-formalism parameters $\beta$ and $\gamma$ (Will 1981) define the gravitational theory chosen for general relativity, $\beta=\gamma=1$. These values have now been confirmed by observation with ever increasing precision and accuracy. The coordinate parameters $\alpha, v$ determine one or another of the most commonly used systems of quasiGalilean coordinates. Denoting the barycentric position vector of the $i$-th body by $\underline{r}_{i}$ and its mass (multiplied by the constant of gravitation) by $M_{i}$, one has

$$
\underline{\rho}_{i}=\underline{r}-\underline{r}_{i}, \underline{r}_{i j}=\underline{r}_{i}-\underline{r}_{j} \text { and } m_{i}=M_{i} / c^{2} \text {. }
$$

The origin of coordinates in Eq. (4) is supposed to coincide with the barycenter of the system of point masses, resulting in

$$
\Sigma_{i} M_{i}\left(1+\frac{1}{2 c^{2}} \underline{t}_{i}^{2}-\frac{1}{2} j \sum_{i} \frac{m_{j}}{r_{i j}}\right) \quad \underline{r}_{i}=0 \text {. }
$$

the transformation to harmonic coordinates $\tilde{\mathrm{t}}$ and $\tilde{\mathfrak{r}}$, which belong to $\alpha=\nu=0$, is given by 
$\left.\tilde{\mathrm{t}}=\mathrm{t}-\frac{\nu}{2 \mathrm{c}^{2}} \sum_{i} \frac{\mathrm{m}_{\mathrm{i}}}{\rho_{\mathrm{i}}} \underline{\underline{\rho}}_{-\mathrm{i}} \underline{\mathbf{r}}_{\mathrm{i}}\right), \quad \tilde{\mathbf{r}}=\underline{\mathbf{r}}-\alpha \sum_{\mathrm{i}} \frac{\mathrm{m}_{\mathrm{i}}}{\rho_{\mathrm{i}}} \rho_{-\mathrm{i}}$.

In particular,

$\tilde{r}_{i j}=r_{i j}-\alpha\left(m_{i}+m_{j}\right)+\alpha \frac{\underline{r}_{i j}}{r_{i j}} \sum_{k \neq i, j} m_{k}\left(\frac{\underline{r}_{j k}}{r_{j k}}-\frac{\underline{r}_{i k}}{r_{i k}}\right)$,

demonstrating the coordinate dependence of the mutual distances between bodies.

The barycentric equations of motion of bodies in the field (4) have the form

$$
\ddot{r}_{i}=-\sum_{j \neq i} \frac{M_{j}}{r_{i j}^{3}} \underline{r}_{i j}+\sum_{j \neq i} m_{j}\left(A_{i j} \underline{r}_{i j}+B_{i j} \underline{s}_{i j}\right) \text {. }
$$

Expressions of $A_{i j}$ and $B_{i j}$ in terms of $\dot{r}_{i}, \underline{r}_{j}, \beta, \gamma$, and $\alpha N$ has no influence on the equations of motion of bodies and light propagation) may be found in Brumberg and Ivanova (1982). On the basis of Eq. (8) it is easy to derive the heliocentric equations of planetary motion: using the index zero on the Sun and denoting by $\underline{r}_{i}=\underline{r}_{i}-\underline{r}_{0}$ the heliocentric position vectors of the planets, we obtain

$$
\begin{aligned}
\underline{R}_{i} & =-\left(M_{0}+M_{i}\right) \frac{\underline{R}_{i}}{R_{i}^{3}}+\sum_{j \neq i} M_{j}\left(\frac{\underline{R}_{j}-\underline{R}_{i}}{r_{i j}^{3}}-\frac{\underline{R}_{j}}{R_{j}^{3}}\right)+ \\
& +\left(m_{0} A_{i o}+m_{i} A_{o i}\right) \underline{R}_{i}+\left(m_{0} B_{i o}+m_{i} B_{o i}\right) \dot{R}_{i}+ \\
& +\sum_{j \neq i} m_{j}\left[A_{i j}\left(\underline{R}_{i}-\underline{R}_{j}\right)+A_{o j} \underline{R}_{j}+B_{i j}\left(\underline{R}_{i}-\underline{R}_{j}\right)+B_{o j} \underline{R}_{j}\right] .
\end{aligned}
$$

Substituting zero as the value of all planetary masses into the relativistic right-hand members of Eq. (9) leads to the Schwarzschild terms. Relativistic perturbations in the motion of the major planets have been investigated in detail by Lestrade and Bretagnon (1982).

With the aid of Eq. (8) applied to the three body problem: Earth, Sun and Moon one may derive the relativistic equations of the Lunar motion in the form adapted for numerical integration (Brumberg and Ivanova 1982). The main relativistic perturbations in the Lunar motion have been found by Lestrade and Chapront-Touze (1982).

For treating the motion of artificial satellites of the Earth such a complete form of the relativistic equations of motion is superfluous, given contemporary standards of accuracy, and it is sufficient to consider in Eq. (4) only the linear combination of the two static Schwarzschild metrics related to the Earth $\left(M_{1}\right)$ and the Sun $\left(M_{2}\right)$. Neglecting $\mathrm{M}_{1} / \mathrm{M}_{2}$ one may introduce the geocentric metric by the transformation 
$\underline{r}=\underline{R}(t)+\underline{\rho}$

with $\underline{\rho}=\underline{\rho}$, and $\underline{R}(t)$ being the heliocentric position vector of the Earth determined by the differential equation of the Schwarzschild problem

$$
\begin{aligned}
& \underline{\ddot{\mathrm{R}}}=-\frac{\mathrm{M}_{2}}{\mathrm{R}^{3}} \underline{\mathrm{R}}+\frac{\mathrm{m}_{2}}{\mathrm{R}^{3}}\left\{\left[-(\alpha+\gamma) \underline{\dot{\mathrm{R}}}^{2}+\frac{3 \alpha}{\mathrm{R}^{2}} \underline{(\mathrm{R}} \underline{\mathrm{R}}\right)^{2}+2(\beta+\gamma-\alpha) \frac{\mathrm{M}_{2}}{\mathrm{R}}\right] \underline{\mathrm{R}}+ \\
& +2(\gamma-\alpha+1)(\underline{\mathrm{R}} \underline{\dot{\mathrm{R}}}) \underline{\mathrm{R}}\} .
\end{aligned}
$$

By virtue of Eq. (10), metric Eq. (4) assumes the following form:

$$
\begin{aligned}
& \mathrm{ds}^{2}=\left\{1-2\left(\frac{\mathrm{m}_{1}}{\rho_{1}}+\frac{\mathrm{m}_{2}}{\rho_{2}}\right)-\frac{1}{\mathrm{c}^{2}} \underline{\mathrm{R}}^{2}+2(\beta-\alpha)\left(\frac{\mathrm{m}_{1}^{2}}{\rho_{1}^{2}}+\frac{\mathrm{m}_{2}^{2}}{\rho_{2}^{2}}\right)+\frac{2 \mathrm{~m}_{2}}{\mathrm{c}^{2} \rho_{2}}\left[(\alpha-\gamma) \underline{\mathrm{R}}^{2}-\right.\right. \\
& \left.\left.-\frac{\alpha}{\rho_{2}^{2}}\left(\underline{\rho}_{2} \underline{\dot{\mathrm{R}}}\right)^{2}\right]\right\rfloor \mathrm{c}^{2} \mathrm{dt}{ }^{2}+\frac{2}{\mathrm{c}}\left\{-\underline{\dot{\mathrm{R}}}+\frac{2 \mathrm{~m}_{2}}{\rho_{2}}\left[(\alpha-\gamma) \underline{\dot{\mathrm{R}}}-\frac{\alpha}{\rho_{2}^{2}}\left(\underline{\rho}_{2} \underline{\dot{\mathrm{R}}}\right) \underline{\rho}_{2}\right]\right\} \underline{\mathrm{\rho}} \cdot \mathrm{cdt}- \\
& -\left[1+2(\gamma-\alpha)\left(\frac{m_{1}}{\rho_{1}}+\frac{m_{2}}{\rho_{2}}\right)\right] d \underline{\rho}^{2}-2 \alpha\left[\frac{m_{1}}{\rho_{1}^{3}}\left(\underline{\rho}_{1} d \underline{\rho}\right)^{2}+\frac{m_{2}}{\rho_{2}^{3}}\left(\underline{\rho}_{2} d \underline{\rho}\right)^{2}\right] .
\end{aligned}
$$

The geocentric equations of motion of artificial satellites of the Earth corresponding to this metric are as follows:

$$
\begin{aligned}
& \ddot{\rho}=-\frac{M_{1}}{\rho^{3}} \underline{\rho}+\frac{M_{2}}{R^{3}}\left[-\underline{\rho}+\frac{3}{R^{2}}(\underline{R} \underline{\rho}) \underline{R}\right]+\ldots+ \\
& +\frac{m_{1}}{\rho^{3}}\left\{\left[-(\alpha+\gamma) \dot{\rho}^{2}+\frac{3 \alpha}{\rho^{2}}(\underline{\rho} \underline{\rho})^{2}+2(\beta+\gamma-\alpha) \frac{M_{1}}{\rho}\right] \underline{\rho}+2(\gamma-\alpha+1)(\underline{\rho} \underline{\rho}) \underline{\rho}\right\}+ \\
& +\frac{2 m_{2}}{\mathrm{R}^{3}}\left\{\left[-(\alpha+\gamma)\left(\underline{\dot{\mathrm{R}}} \dot{\rho}+\frac{1}{2} \underline{\dot{\rho}}^{2}\right)+\frac{3 \alpha}{\mathrm{R}^{2}}(\underline{\mathrm{R}} \underline{\dot{\mathrm{R}}})(\underline{\mathrm{R}} \underline{\dot{\rho}})+\frac{1}{2}(\underline{\mathrm{R}} \underline{\dot{\rho}})^{2}\right)\right] \underline{\mathrm{R}}+ \\
& +(\gamma-\alpha+1)(\underline{\mathrm{R}} \underline{\dot{\rho}}) \underline{\mathrm{R}}+(\gamma-\alpha+1)[(\underline{\mathrm{R}} \underline{\mathrm{R}})+(\underline{\mathrm{R}} \underline{\underline{\rho}})] \underline{\dot{\rho}}\}+\ldots .
\end{aligned}
$$

In the relativistic right-hand members of Eq. (13), the terms related to the combined action of the Earth and the Sun have been neglected. Besides this the solar part in Eq. (13) 
contains only the terms remaining in the limit for $\rho / R \rightarrow 0$. Therefore, Eq. (13) describes three kinds of the relativistic perturbations: 1) indirect solar perturbations caused by substituting the relativistic value $R(t)$ from Eq. (11) into the Newtonian right-hand members of Eq. (13); 2) the direct Schwarzschild perturbations due to the Earth; 3) the direct solar perturbations. It may be noted that in the model of the circular heliocentric motions of the Earth with $\underline{\mathrm{RR}}=0$ the solar terms in Eq. (13) are rewritten in the form

$$
(\delta \underline{\rho})_{\text {sol }}=\frac{m_{2}}{\mathrm{R}^{3}}\left\{(2 \gamma+1)[\underline{\dot{\rho}} \times(\underline{\dot{R}} \times \underline{\mathrm{R}})]+(1-2 \alpha)[(\underline{\dot{R}} \underline{\dot{\rho}}) \underline{\mathrm{R}}+(\underline{\mathrm{R}} \underline{\dot{\rho}}) \underline{\dot{\mathrm{R}}}]+0\left(\underline{\rho}^{2}\right)\right\} \text {. }
$$

The first term in Eq. (14) describes the geodesic precession resulting in secular motions of the perigee and the node (1.91"per century). The second term leads to the periodic perturbations, and those terms of Eq. (13) which are quadratic in the geocentric velocities are designated by $0\left(\dot{\rho}^{2}\right)$.

The time argument in Egs. (12) and (13) is the coordinate time $t$ of the barycentric system Sun-Earth which coincides with the heliocentric coordinate time when $M_{1} / M_{2}$ is neglected. In some recent papers (for example: Ashby and Bertotti 1983, 1984) the satellite motion is treated in the local inertial geocentric reference frame with geocentric coordinate time being an independent argument. Such an approach provides a significant simplification of the solar part of the relativistic terms. Yet it seems desirable to have one and the same argument in the dynamical theories of motion of natural as well as artificial bodies of the Solar system. From this point of view the approach based on Eqs. (12) and (13) with the barycentric coordinate time as an argument may be more straightforward.

\section{General technique of the relativistic reduction in astrometry}

Relativistic reduction in astrometry may be reduced to the solution of several typical problems, each of them having individual meaning.

The first problem is the transformation of an observer's proper time $\tau$ to the coordinate time $t$ of the barycentric reference frame. This is performed by integrating the equation

$$
d \tau=\left(g_{o o}+\frac{2}{c} g_{o i} \dot{x}^{i}+\frac{1}{c^{2}} g_{i j} \dot{x}^{i} \dot{x}^{j}\right)^{1 / 2} d t
$$

with $\dot{x}$ being the components of the observer's barycentric velocity. The basic relationship between $\tau$ and $t$ was treated in detail by Moyer (1981). The more delicate relations between International Atomic Time, Barycentric Dynamical Time and Terrestrial Dynamical Time are discussed by Aoki et al. (1982). It should be remembered that the last two arguments are, of course, coordinate-dependent.

The second problem is to investigate the light propagation in the field Eq. (1) using the same coordinates as in the dynamical problem under consideration. Integration of the equations of light propagation 


$$
\begin{aligned}
\ddot{\mathrm{x}} & =-\frac{1}{2} c^{2} h_{o o, i}+h_{o o, k} \dot{x}^{k} \dot{x}^{i}+\left(h_{i k, l}-\frac{1}{2} h_{k l, i}\right) \dot{x}^{k} \dot{x}^{l}+ \\
& +c\left(h_{o i, k}-h_{o k, i}\right) \dot{x}^{k}+\frac{1}{c} h_{o k, j} \dot{x}^{j} \dot{x}^{k} \dot{x}^{i}
\end{aligned}
$$

yields the value of the coordinate velocity $(t) / c$ at the point of observation. As an initial value one may take, for example, the unit vector $\sigma=\hat{i}(-\infty) / c, \sigma^{2}=1$, characterizing the initial direction of the light at infinity. For the boundary value problem $\underline{r}_{0}\left(t_{0}\right)=\underline{r}_{0}$, $\underline{r}\left(t_{1}\right)=\underline{r}_{1}$ one has to calculate $\underline{\sigma}$ and $t_{1}-t_{0}$ in terms of these boundary values.

The measurement of the angular distance between two light emitters may be regarded as the basic idealized type of astrometric observations. Therefore, the third problem of a relativistic reduction is to calculate an expression for the angle between two light rays at the point of observation. For this purpose one may apply the local splitting of Eq. (1) at the point of observation as follows

$$
\begin{aligned}
& d s^{2}=c^{2} d \tau^{2}-d l^{2} \\
& c d \tau=\sqrt{g_{o o}}\left(d x^{o}+\frac{1}{g_{o o}} g_{o i} d x^{i}\right), \\
& d l^{2}=\gamma_{i j} d x^{i} d x^{j} ; \gamma_{i j}=\frac{1}{g_{o o}} g_{o i} g_{o j}-g_{i j} .
\end{aligned}
$$

The three-dimensional metric form $\mathrm{dl}^{2}$ describes the local space relations at the point of observation. Scalar product and length, respectively, of arbitrary threedimensional vectors applied at the point of observation are defined by the formulas

$$
(\underline{P})_{r e l}=\gamma_{i j} P^{i} Q^{j}, \quad P_{r e l}=\left(\gamma_{i j} P^{i} P^{j}\right)^{1 / 2}
$$

thus yielding the following expression for the angle between these vectors

$$
\cos \psi=\frac{\left(\underline{\mathrm{P}} \underline{Q}_{\text {rel }}\right.}{\mathrm{P}_{\text {rel }} \mathrm{Q}_{\text {rel }}} .
$$

If $P, Q$ are identified with the directions of two light rays at the point of observation, then $\underline{P}=\underline{\dot{r}}_{1}(t) / c, \underline{Q}=\underline{\dot{r}}_{2}(t) / c$ and the relation Eq. (21) will represent the required expression for the measurable angular distance between two light emitters.

When actually discussing the results of an observation, one usually deals with the angular distances between celestial bodies and the relativistic reduction based on Eq. (21) may be quite adequate. Nevertheless, astrometric practice of ten requires the derivation of the coordinates of a star or a planet with respect to some specified reference system. 
the forth problem of reduction is thus to calculate the actually measurable position of an emitter in the reference frame of an observer.

The reference frame of an observer may be mathematically represented by the tetrad of unit orthogonal vectors $\lambda(\mu), \mu=0,1,2,3$. The time-like vector $\lambda(0)$ is tangent to the world-line of the observer at the point of observation. Physically, this vector determines the proper time $d \tau$ of the reference frame. The three other yectors $\lambda_{(i)}(i=$ $1,2,3)$ are space-like and define three space directions in the space $\mathrm{dl}^{2}$. This tetrad represents the local inertial reference system at the point of observation. All relations of special relativity are locally valid in such a system and all tensor operations are carried out with the aid of $\eta_{n}$. The tetrad is completely determined by 16 scalar components $\lambda(\mu)$. Changing the local and global indices one gets

$$
\lambda_{\alpha}^{(\beta)}=\eta^{\beta \mu} g_{\alpha \nu} \lambda \stackrel{\nu}{\nu}
$$

From this follows

$$
g_{\mu \nu}=\lambda \underset{\mu}{(\alpha)} \lambda_{\nu}^{(\beta)} \eta_{\alpha \beta}
$$

and

$$
d s^{2}=\eta_{\mu \nu} d x^{(\mu)} d x^{(\nu)}
$$

with

$$
d x^{(\mu)}=\lambda \underset{\nu}{(\mu)} d x^{\nu}, d x^{\mu}=\lambda{ }_{(\nu)}^{\mu} d x^{(\nu)}
$$

Relations Egs. (22)-(25) are basic for the tetrad formalism (Ivanitskaya 1979).

For the metric Eq. (17) one has in accordance with the general form Eqs. (1), (2)

$$
h_{o o}=0, h_{o i}=0, h_{i j}=\delta_{i j}-\gamma_{i j}
$$

and the tetrad components may be found from

$$
\lambda \underset{(\nu)}{\mu}=\delta_{\nu}^{\mu}-\frac{1}{2} \eta^{\mu \alpha} h_{v a} \text {. }
$$

The results are 


$$
\lambda \underset{(0)}{\stackrel{o}{o}}=1, \lambda \underset{(0)}{i}=0, \lambda \underset{(j)}{\stackrel{o}{j}}=0, \lambda \underset{(j)}{i}=\delta_{i j}+\frac{1}{2} h_{i j}
$$

and

$$
\lambda_{0}^{(0)}=1, \lambda_{i}^{(0)}=0, \lambda_{0}^{(j)}=0, \lambda_{i}^{(j)}=\delta_{i j}-\frac{1}{2} h_{i j} .
$$

The 4-impulse of a photon has the following components in metric Eq. (17)

$$
p^{0}=h \nu, p^{i}=\frac{h \nu}{c} \frac{d x^{i}}{d \tau}
$$

( $\nu$ is the frequency, $h$ is Planck's constant). The components $p^{i}$ may be calculated on the basis of the equations of light transmittion in field Eq. (1) with the use of Eq. (18). Then the tetrad formalism enables one to find the invariant components of the 3-vector

$$
\begin{aligned}
& \mathrm{p}=\left(\mathrm{p}^{(1)}, \mathrm{p}^{(2)}, \mathrm{p}^{(3)}\right) \\
& \mathrm{p}^{(\mathrm{i})}=\mathrm{p}_{\lambda}^{\mu}{ }_{\mu}^{(\mathrm{i})} .
\end{aligned}
$$

With the normalization $h \nu=1$, vector $p$ represents the unit vector determining the observed direction of the light ray at the point of observation. Relation Eq. (31) may be rewritten in the form

$$
\underline{\sigma}=\underline{p}+\Delta \underline{p}
$$

The correction $\Delta \rho$ represents the relativistic term which must be added to the observed value of $\mathrm{p}$ in order to obtain the gravitationally unperturbed direction $\underline{\sigma}$ at infinity.

It is easy to express this reduction in spherical coordinates. For example, using equatorial coordinates

$$
\mathrm{p}=-(\cos \alpha \cos \delta, \sin \alpha \cos \delta, \sin \delta)
$$

one has

$$
\cos \delta \Delta \alpha=\sin \alpha \Delta p^{1}-\cos \alpha \Delta p^{2}+\sin \delta \Delta \alpha \Delta \delta,
$$


$\Delta \delta=\sin \delta\left(\cos \alpha \Delta \mathrm{p}^{1}+\sin \alpha \Delta \mathrm{p}^{2}\right)-\cos \delta \Delta \mathrm{p}^{3}-\frac{1}{2} \sin \delta \cos \delta(\Delta \alpha)^{2}$.

It should be noted that the expression for the vector $p$ may be obtained quite formally with the aid of Eq. (17). Starting from the expansion $\mathrm{dl}^{2}=\mathrm{dr}^{2}+\ldots$ one derives the vector $\mathrm{dl}=\mathrm{dr}+\ldots$ (ellipses indicate the relativistic terms) as well as the derivative $\mathrm{d} \underline{\mathrm{l}} / \mathrm{d} \tau$, using $\underline{\mathbf{r}}(\mathrm{t})$ for the light. Then,

$\mathrm{p}=\frac{1}{\mathrm{c}} \frac{\mathrm{d} \underline{\mathrm{l}}}{\mathrm{d} \tau}$.

Actually this method has been used by Murray (1981), and the tetrad formulation Eq. (31) may be regarded as its foundation.

The angle between two light rays from the directions $\mathrm{p}_{1}, \mathrm{p}_{2}$, respectively, at the point of observation is defined by the Euclidean formula

$\cos \psi=\mathrm{p}_{1} \cdot \mathrm{p}_{2}$

which is equivalent to Eq. (21).

The fifth problem of relativistic reduction is to take into account the observer's motion. This may be solved by using the formulas for the relativistic treatment of aberration independently of the previous problems. One may achieve the same goal, however, by substituting into Eq. (1) the expression Eq. (10) with $\mathrm{R}(\mathrm{t})$ being that function of time which defines the motion of the observer (for example, annual and diurnal motion of the Earth). If all this is done, one gets expressions which describe the dependence of $\cos \psi$ and $p$ not only on the parameters of the graitational field but on the observer's velocity $\underline{\dot{R}}(\mathrm{t})$ as well.

The last problem to be mentioned here is the relativistic treatment of the astronomical reference frames. To construct a co-moving system suited for the reference frame, Eq. (17) must be divided not at one world-point but along the whole world-line of the observer in some space tube of this line. Such problems may be solved by the methods of the tetrad formalism (Mast and Strathdee 1959; Synge 1960; Manasse and Misner 1963). The physical aspects of the relativistic reference frames are considered by Moller (1972) and Vladimirov (1982). The construction of the reference systems on the basis of given dynamical relations or space directions (prototype of dynamical and kinematical reference frames in astronomy) was dealt with by Vyblyj and Kostyukovich (1982) and by Kostyukovich (1982). Relations among barycentric, geocentric and topocentric reference systems are discussed by Fujimoto et al. (1982) and by Pavlov (1984).

\section{Relativistic reduction in the Schwarzschild problem and in the two-body field}

Consider now some applications of the general technique of relativistic reduction.

In the most simple case of the heliocentric Schwarzschild metric one has 


$$
\begin{aligned}
& h_{o o}=-\frac{2 m}{r}+2[\beta-\alpha(r)] \frac{m^{2}}{r^{2}}+\ldots, \quad h_{o i}=0, \\
& h_{i j}=-\frac{2 m}{r}\left\{[r-\alpha(r)] \delta_{i j}+\left[\alpha(r)-r \alpha^{\prime}(r)\right] \frac{x^{i} x^{j}}{r^{2}}\right\}+\ldots
\end{aligned}
$$

where the coordinate function $\alpha(r)$ generalizes the constant coordinate parameter $\alpha$ and satisfies two relations for the quasi-Galilean coordinates $\alpha^{\prime}(r) \rightarrow 0, \alpha(r) / r \rightarrow 0$ with $r \rightarrow \infty$.

By virtue of Eq. (12) the velocity of light in the field Eq. (38) will be

$$
\begin{aligned}
& \frac{1}{c} \underline{r}(t)=\underline{\sigma}+\frac{m}{r}\left\{\left[r \alpha^{\prime}(r)-\alpha(r)\right] \frac{(\underline{\sigma})}{r^{2}} \underline{r}+\right. \\
& +[\alpha(r)-\gamma-1] \underline{\sigma}-(\gamma+1) \frac{\underline{\sigma} \times(\underline{r} \times \underline{\sigma})}{r-\underline{\sigma} \underline{r}} \mid .
\end{aligned}
$$

For the boundary value problem $\underline{r}\left(t_{0}\right)=\underline{r}_{0}, \underline{r}\left(t_{1}\right)=\underline{r}_{1}$ one obtains

$$
\begin{aligned}
& \underline{\sigma}=\frac{\underline{D}_{01}}{\mathrm{D}_{01}}+\frac{\mathrm{m}}{\mathrm{D}_{01}}\left\{(\gamma+1) \frac{\mathrm{r}_{1}-\mathrm{r}_{0}+\mathrm{D}_{01}}{\left(\underline{\mathrm{r}}_{0} \times \underline{\mathrm{r}}_{1}\right)^{2}}+\right. \\
& \left.+\frac{1}{\mathrm{D}_{01}^{2}}\left[\frac{\alpha\left(\mathrm{r}_{0}\right)}{\mathrm{r}_{0}}-\frac{\alpha\left(\mathrm{r}_{1}\right)}{\mathrm{r}_{1}}\right]\right\}\left[\underline{D}_{01} \times\left(\underline{r}_{0} \times \underline{r}_{1}\right)\right], \\
& \mathrm{c}\left(\mathrm{t}_{1}-\mathrm{t}_{0}\right)=\mathrm{D}_{01}+\mathrm{m}\left\{(\gamma+1) \mathrm{l}_{\mathrm{n}} \frac{\mathrm{r}_{0}+\mathrm{r}_{1}+\mathrm{D}_{01}}{\mathrm{r}_{0}+\mathrm{r}_{1}-\mathrm{D}_{01}}-\right. \\
& \left.-\alpha\left(\mathrm{r}_{1}\right) \frac{\mathrm{r}_{1}^{2}-\mathrm{r}_{0}^{2}+\mathrm{D}_{01}^{2}}{2 \mathrm{r}_{1} \mathrm{D}_{01}}+\alpha\left(\mathrm{r}_{0}\right) \frac{\mathrm{r}_{1}^{2}-\mathrm{r}_{0}^{2}-\mathrm{D}_{0}^{2}}{2 \mathrm{r}_{0} \mathrm{D}_{01}}\right\},
\end{aligned}
$$

where $\underline{\mathrm{D}}_{01}=\underline{\mathrm{r}}_{1}-\underline{\mathrm{r}}_{0}$. For the case $\mathrm{r} \ll \mathrm{r}_{0}$ it is convenient to use the aproximate relations

$$
\underline{\sigma}=-\frac{\underline{r}_{0}}{\mathrm{r}_{0}}+\left\{1+\frac{\mathrm{m}}{\mathrm{r}_{1}}\left[\frac{\gamma+1}{1+\frac{\underline{r}_{0} \underline{r}_{1}}{\mathrm{r}_{0} \mathrm{r}_{1}}}-\alpha\left(\mathrm{r}_{1}\right)\right]\right\} \frac{\left[\underline{\mathrm{r}}_{0} \times\left(\underline{\mathrm{r}}_{1} \times \underline{\mathrm{r}}_{0}\right)\right]}{\mathrm{r}_{0}^{3}},
$$




$$
\begin{aligned}
& c\left(t_{1}-t_{0}\right)=r_{0}\left(1-\frac{\underline{r}_{0}-r_{1}}{2}\right)+m\left[(\gamma+1) \ln \frac{2 r_{0}^{2}}{r_{0}^{2} r_{1}+\underline{r}_{0} \underline{r}_{1}}+\right. \\
& \left.+\frac{\underline{r}_{0} \underline{r}_{1}}{r_{0} r_{1}} \alpha\left(r_{1}\right)-\alpha\left(r_{0}\right)\right] .
\end{aligned}
$$

The function $\alpha(r)$ enters into the right-hand sides of Eqs. (40) and (41) only through the combination

$$
\tilde{r}=\underline{r}\left[1-\frac{m}{r} \alpha(r)\right] \text {, }
$$

proving the coordinate independence of $\sigma$ and $t_{1}-t_{0}$ (taking into account that in the postNewtonian approximation the relationship between proper time and coordinate time is coordinate independent).

The geocentric Schwarzschild metric is introduced with the gid of Eq. (10). If the metric tensor Eq. (38) in the heliocentric system is designated by $h_{\mu \nu}^{*}$, then one has in the geocentric system

$$
\begin{aligned}
& h_{o o}=h_{o o}^{*}-\frac{1}{c^{2}} \dot{R}^{2}-\frac{2 m}{c^{2} r}[\gamma-\alpha(r)] \underline{R}^{2}-\frac{2 m}{c^{2} r^{3}}\left[\alpha(r)-r \alpha^{\prime}(r)\right](\underline{r} \underline{\dot{R}})^{2}, \\
& h_{o i}=-\frac{1}{c}\left\{\dot{R}^{i}+\frac{2 m}{r}[\gamma-\alpha(r)] \dot{R}^{i}+\frac{2 m}{r^{3}}\left[\alpha(r)-r \alpha^{\prime}(r)\right](\underline{r} \dot{R}) x^{i}\right\}, \\
& h_{i j}=h_{i j}^{*} .
\end{aligned}
$$

In this metric, Eq. (17) is split in the following way:

$$
\begin{aligned}
& \mathrm{dl}^{2}=\left\{1+\frac{2 \mathrm{~m}}{\mathrm{r}}[\gamma-\alpha(\mathrm{r})]\right\} \mathrm{d}^{2}+\frac{2 \mathrm{~m}}{\mathrm{r}^{3}}\left[\alpha(\mathrm{r})-\mathrm{r} \alpha^{\prime}(\mathrm{r})\right]\left(\underline{\mathrm{r}} \mathrm{d} \underline{)^{2}}+\frac{1}{\mathrm{c}^{2}}\left(\underline{\mathrm{R}} \mathrm{d} \underline{{ }^{2}}\right)^{2},\right. \\
& \mathrm{d} \tau=\left\{1-\frac{\mathrm{m}}{\mathrm{r}}-\frac{1}{2 \mathrm{c}^{2}} \underline{\mathrm{R}}^{2}+\left[\beta-\alpha(\mathrm{r})-\frac{1}{2}\right] \frac{\mathrm{m}^{2}}{\mathrm{r}^{2}}-\frac{\mathrm{m}}{\mathrm{c}^{2} \mathrm{r}}\left[\gamma-\alpha(\mathrm{r})+\frac{1}{2}\right] \underline{\mathrm{R}}^{2}-\right. \\
&-\frac{\mathrm{m}}{\mathrm{c}^{2} \mathrm{r}^{3}}\left[\alpha(\mathrm{r})-\mathrm{r} \alpha^{\prime}(\mathrm{r})\right](\underline{\mathrm{r}} \underline{\mathrm{R}})-\frac{1}{8 \mathrm{c}^{4}}\left(\underline{\mathrm{R}}^{2}\right)^{2}\left|\mathrm{dt}-\frac{1}{\mathrm{c}^{2}}\right| 1+\frac{2 \mathrm{~m}}{\mathrm{r}}\left[\gamma-\alpha(\mathrm{r})+\frac{1}{2}\right]+
\end{aligned}
$$




$$
+\frac{1}{2 c^{2}} \mid \underline{\mathrm{R}}^{2}(\underline{\dot{R}} \mathrm{~d} \underline{\rho})-\frac{2 \mathrm{~m}}{\mathrm{c}^{2} \mathrm{r}^{3}}\left[\alpha(\mathrm{r})-\mathrm{r} \alpha^{\prime}(\mathrm{r})\right](\underline{\mathrm{r}} \underline{\dot{\mathrm{R}}}) \quad(\underline{\mathrm{r}} \mathrm{d} \underline{\rho}) .
$$

With the aid of Eqs. (10), (39) and (45) the relation Eq. (21) results in the final expression for the angle between two light rays (Brumberg 1981a)

$$
\begin{aligned}
& \cos \psi=\underline{\sigma}_{1} \underline{\sigma}_{2}+\frac{1}{\mathrm{c}}\left(\underline{\sigma}_{1} \underline{\sigma}_{2}-1\right)\left(\underline{\sigma}_{1} \underline{\dot{\mathrm{R}}}+\underline{\sigma}_{2} \underline{\dot{\mathrm{R}}}\right)+\frac{1}{\mathrm{c}^{2}}\left(\underline{\sigma}_{1} \underline{\sigma}_{2}-1\right)\left[\left(\underline{\sigma}_{1} \underline{\dot{\mathrm{R}}}\right)^{2}+\left(\underline{\sigma}_{2} \underline{\dot{\mathrm{R}}}\right)^{2}+\right. \\
& \left.+\left(\underline{\sigma}_{1} \underline{\dot{\mathbf{R}}}\right)\left(\underline{\sigma}_{2} \underline{\dot{\mathbf{R}}}\right)-\underline{\mathbf{R}}^{2}\right]+(\gamma+1) \frac{\mathrm{m}}{\mathbf{r}}\left(\frac{\underline{\mathbf{r}} \times \underline{\sigma}_{1}}{\mathbf{r}-\underline{\mathbf{r}}_{-}}-\frac{\underline{\mathbf{r}} \times \underline{\sigma}_{2}}{\mathbf{r}-\underline{\mathbf{r}} \underline{\sigma}_{2}}\right)\left(\underline{\sigma}_{1} \times \underline{\sigma}_{2}\right) .
\end{aligned}
$$

This formula describes the aberration effects of the first and second order together with the light deflection effect.

Applying to Eqs. (45) and (46) the tetrad relations Eqs. (27)-(29) and using Eq. (31), one gets an expression for the proper direction of the light at the point of observation

$$
\begin{aligned}
& \mathrm{p}=\underline{\sigma}+\frac{1}{\mathrm{c}}[\underline{\sigma} \times(\underline{\sigma} \times \underline{\dot{R}})]-\frac{1}{2 \mathrm{c}^{2}}[\underline{\dot{R}} \times(\underline{\sigma} \times \underline{\dot{R}})]+\frac{1}{\mathrm{c}^{2}}(\underline{\sigma} \underline{\dot{R}})[\underline{\sigma} \times(\underline{\sigma} \times \underline{\dot{R}})]- \\
& -(\gamma+1) \frac{\mathrm{m}}{\mathrm{r}} \frac{\underline{\sigma} \times(\underline{\mathrm{r}} \times \underline{\sigma})}{\mathrm{r}-\underline{\sigma} \underline{\mathbf{r}}} .
\end{aligned}
$$

This expression may also be obtained directly from Eq. (36) with the aid of Eqs. (45) and (46). In spite of the difference in form, the expression Eq. (48) agrees with the corresponding relation given by Murray (1981). field

Solving Eq. (48) with respect to $\underline{\sigma}$ gives the reduction Eq. (32) in the Schwarzschild

$$
\begin{aligned}
\Delta \mathrm{p} & =\frac{1}{\mathrm{c}}[\mathrm{p} \times(\underline{\dot{R}} \times \underline{\mathrm{p}})]-\frac{1}{\mathrm{c}^{2}}(\underline{\dot{R}} \times \underline{\mathrm{p}})^{2} \mathrm{p}-\frac{1}{2 \mathrm{c}^{2}}[\underline{\dot{\mathrm{R}}} \times(\underline{\dot{\mathrm{R}}} \times \underline{\mathrm{p}})]+ \\
& +(\gamma+1) \frac{\mathrm{m}}{\mathrm{r}} \frac{\frac{\mathrm{p} \times(\underline{\mathrm{r}} \times \underline{\mathrm{p}})}{\mathrm{r}-\underline{\mathrm{p}} \underline{\mathrm{r}}} .}{} .
\end{aligned}
$$

Using Eq. (47), one may investigate a variety of specific cases occuring in astrometric practice (Brumberg 1981b). As illustration, we consider here the relativistic corrections in defining the annual parallax. This question has aroused some controversy (Arifov and Kadyev 1968; Mikhailov 1969; Arifov 1983).

In principle, the distance to the star whose heliocentric position vector is $\underline{\mathbf{r}}_{0}$ may be 
found by two properly spaced measurements of the angle between the directions from the Earth to the Sun and to the star. Neglecting aberration effects and using Eq. (42), one finds from Eq. (47) for the first observation $\phi_{1}$ when the heliocentric position vector of the Earth is $\underline{\mathbf{r}}_{1}$

$$
\begin{aligned}
& \cos \phi_{1}+\frac{\underline{r}_{1} \underline{r}_{0}}{r_{1} r_{0}}+\frac{\left|\underline{r}_{1} \times \underline{r}_{0}\right|^{2}}{r_{1} r_{0}^{3}}+m\left(\frac{\underline{r}_{1} \underline{r}_{0}}{r_{1} r_{0}}-1\right)\left[\frac{\gamma+1}{r_{1}}+\left(\frac{\underline{r}_{1}-\underline{r}_{0}}{r_{1} r_{0}}+1\right) \frac{\alpha\left(r_{1}\right)}{r_{0}}\right] \\
& \sin \phi_{1}=-\frac{\left|\underline{r}_{0} \times \underline{r}_{1}\right|}{\mathbf{r}_{0} \mathbf{r}_{1}}\left\{1+\frac{\underline{r}_{1} \underline{r}_{0}}{\mathbf{r}_{0}^{2}}-m\left[\frac{\underline{r}_{1} \underline{r}_{0}}{r_{1} \mathbf{r}_{0}+\underline{r}_{1} \underline{r}_{0}} \frac{\gamma+1}{r_{1}}+\frac{\underline{r}_{1} \underline{r}_{0}}{r_{1} r_{0}} \frac{\alpha\left(r_{1}\right)}{r_{0}}\right]\right. \text {. }
\end{aligned}
$$

At the moment of the second observation $\phi_{2}$ one has the same relations but with the sign of $\underline{\mathbf{r}}_{1}$ reversed, provided that $\phi_{1}$ and $\phi_{2}$ were measured one half year apart. As a consequence, we have

$$
\frac{\sin \left(\phi_{1}+\phi_{2}\right)}{2 \sin \phi_{1}}=\frac{r_{1}-m \alpha\left(r_{1}\right)}{r_{0}}-(\gamma+1) \frac{m}{r_{1} \sin ^{2} \phi_{1}} .
$$

The classical definition specifies as the annual parallax the angle $\Delta$ at the star in the rectangular triangle Sun-Earth-star with $\phi_{1}=90^{\circ}$. In Newtonian theory, $\phi_{2}=90^{\circ}-2 \Delta$ and the left-hand side of Eq. (52) becomes equal to $\Delta$. Starting from some sufficiently large value of $r_{0}$, the right-hand side of Eq. (52) will be negative. But the conclusion about the negative value of $\Delta$ would be incorrect being based on the Euclidean formula for the sum of angles in the triangle. Assuming that the Earth moves uniformly in a circle with the radius $\mathrm{r}_{1}=\mathrm{a}$ and the mean motion

$$
\mathrm{n}=\left(\mathrm{M} / \mathrm{a}^{3}\right)^{1 / 2}\left\{1+\frac{\mathrm{m}}{\mathrm{a}}\left[\frac{3}{2} \alpha(\mathrm{a})-\beta-\frac{1}{2} \gamma\right]\right\}
$$

one has

$a=a^{N}+\Delta a, a^{N}=\left(M / n^{2}\right)^{1 / 3}$,

where $a^{N}$ is (by optical means or by radar) a measurable quantity with the difference $\Delta \mathbf{a}-$ $\mathrm{ma}(\mathrm{a})$ being coordinate-independent. In principle, the relation Eq. (52) permits one to find the ratio $a^{\mathrm{N}} / \mathrm{r}_{0}$ without any ambiguities.

Consider now the modification of the basic reduction formulas for the field Eq. (12) of the two-body system Earth-Sun. The barycentric velocity of light will be 


$$
\frac{1}{\mathbf{c}} \underline{\dot{r}}(t)=\underline{\sigma}+\sum_{i=1}^{2} \frac{m_{i}}{\rho_{i}}\left[-\alpha \frac{\underline{\sigma} \rho_{i}}{\rho_{i}^{2}} \varrho_{i}+(\alpha-\gamma-1) \underline{\sigma}-(\gamma+1) \frac{\underline{\sigma} \times\left(\underline{\rho}_{i} \times \underline{\sigma}\right)}{\rho_{i}-\underline{\sigma} \rho_{i}}\right]
$$

and relations Eqs. (40) and (41) for the boundary value problem take the form

$$
\begin{aligned}
& \underline{\sigma}=\frac{\underline{D}_{01}}{D_{01}}+\sum_{i=1}^{2} \frac{m_{i}}{D_{01}}\left\{(\gamma+1) \frac{\rho_{i}^{(1)}-\rho_{i}^{(0)}+D_{01}}{\left(\rho_{i}^{(0)} \times \rho_{i}^{(1)}\right)^{2}}+\frac{\alpha}{D_{01}^{2} \rho_{i}^{(0)} \rho_{i}^{(1)}}\left(\rho_{i}^{(1)}-\rho_{i}^{(0)}\right) \mid x\right. \\
& \times\left[\underline{D}_{01} \times\left(\varrho_{i}^{(0)} \times \varrho_{i}^{(1)}\right)\right], \\
& c\left(t_{1}-t_{0}\right)=D_{01}+\sum_{i=1}^{2} m_{i}\left\{(\gamma+1) \ln \frac{\rho_{i}^{(1)}+\rho_{i}^{(0)}+D_{01}}{\rho_{i}^{(1)}+\rho_{i}^{(0)}-D_{01}}+\right. \\
& \left.+\frac{\alpha}{2 D_{01} \rho_{i}^{(0)} \rho_{i}^{(1)}}\left(\rho_{i}^{(1)}+\rho_{i}^{(0)}\right)\left[\left(\rho_{i}^{(1)}-\rho_{i}^{(0)}\right)^{2}-D_{01}^{2}\right]\right] \text {. }
\end{aligned}
$$

Here, $\rho_{i}^{(j)}$ denotes the position vector of the light particle with respect to the body $i$ at the moment j. Eq. (17) is partitioned for the metric Eq. (12) as follows:

$$
\begin{aligned}
& \mathrm{dl}^{2}=\left[1+2(\gamma-\alpha) \underset{\mathrm{i}=1}{2} \frac{\mathrm{m}_{\mathrm{i}}}{\rho_{\mathrm{i}}}\right] \mathrm{d}^{2}+\frac{1}{\mathrm{c}^{2}} \quad(\underline{\mathrm{R}} \mathrm{d} \underline{\mathrm{L}})^{2}+2 \alpha \underset{\mathrm{i}=1}{2} \frac{\mathrm{m}_{\mathrm{i}}}{\rho_{\mathrm{i}}} \quad\left(\underline{\rho}_{\mathrm{i}} \mathrm{d} \underline{\rho}\right)^{2}, \\
& \left.\mathrm{~d} \tau=1-\sum_{\mathrm{i}=1}^{2} \frac{\mathrm{m}_{\mathrm{i}}}{\rho_{\mathrm{i}}}-\frac{1}{2 \mathrm{c}^{2}} \underline{\mathrm{R}}^{2}+\left(\beta-\alpha-\frac{1}{2}\right) \sum_{\mathrm{i}=1}^{2} \frac{\mathrm{m}_{\mathrm{i}}^{2}}{\rho_{\mathrm{i}}^{2}}-\frac{\mathrm{m}_{1} \mathrm{~m}_{2}}{\rho_{1} \rho_{2}}-\frac{1}{8 \mathrm{c}} \underline{\mathrm{R}}^{2}\right)^{2}- \\
& \left.-\frac{\mathrm{m}_{1}}{2 \mathrm{c}^{2} \rho_{1}} \stackrel{.}{\mathrm{R}}^{2}+\frac{\mathrm{m}_{2}}{\mathrm{c}^{2} \rho_{2}}\left[\left(\alpha-\gamma-\frac{1}{2}\right) \underline{\mathrm{R}}^{2}-\frac{\alpha}{\rho_{2}^{2}}\left(\underline{\rho}_{2} \underline{\mathrm{R}}^{2}\right)^{2}\right]\right] \mathrm{dt}+ \\
& +\frac{1}{c^{2}}\left\{\left[-1-\frac{m_{1}}{\rho_{1}}+(2 \alpha-2 \gamma-1) \frac{m_{2}}{\rho_{2}}-\frac{1}{2 c^{2}}\right] \underline{\dot{R}}^{2}-2 \alpha \frac{m_{2}}{\rho_{2}}\left(\underline{\rho}_{2} \underline{\dot{R}}\right) \underline{\rho}_{2} \mid d_{\varrho}\right. \text {. }
\end{aligned}
$$

For the Earth-Sun system, formulas Egs. (47), (48) are generalized to 


$$
\begin{aligned}
& \cos \psi=\sigma_{-1} \sigma_{-2}+(\gamma+1) \sum_{i=1}^{2} \frac{m}{\rho}_{i} \frac{\rho_{-i} \times \sigma_{-1}}{\rho_{i}-\rho_{-i} \sigma_{-1}}- \\
& \left.-\frac{\rho_{-i} \times \sigma_{-2}}{\rho i-\rho_{-i}-2}\right)\left(\sigma_{-1} \times-2\right)+\ldots \text {, }
\end{aligned}
$$

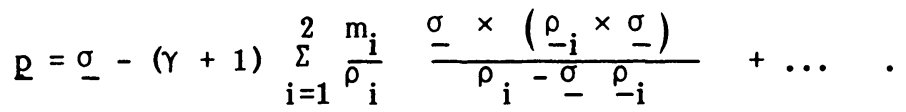

The ellipses in Eqs. (57) and (58) signify the aberration terms which are the same as in Eqs. (47) and (48), respectively.

With the aid of Eqs. (56) and (59), one may derive the round-trip time in Lunar laser ranging as well as the expressions for the angular distances Moon-Sun and Moon-star (Brumberg and Ivanova 1982).

\section{Relativistic reduction in geodynamies}

As supplementary examples, the relativistic reduction in Doppler satellite observations, navigation measurements with the use of satellites like NAVSTAR and radiointerferometric observations may be considered.

In the first two problems one may (for a precision of $10^{-14}$ in the frequency measurements) neglect the influences of the Moon and the Sun (see appendix) and consider the geocentric inertial system Eq. (1) as characterized by

$$
h_{o o}=2^{\Phi}(\underline{r}) / c^{2}, \quad h_{o i}=0, h_{i j}=0 \text {, }
$$

$\Phi(\underline{r})$ being the Newtonian potential at $\underline{r}$. If the moving point 1 emits a light signal of duration $\delta_{t}$ and the duration of this signal as received at the moving point 2 is $\delta^{\prime}$, then the ratio of the emitted frequency $f_{1}$ to the received frequency $f_{2}$ will be in inverse proportion to the ratio of the proper time intervals $\delta_{t} / \delta_{t^{\prime}}$ of light emission and reception. Therefore

$$
\frac{\mathrm{f}_{1}}{\mathrm{f}_{12}} \equiv \frac{\delta \tau^{\prime}}{\delta \tau}=\frac{1+\Phi_{2} / \mathrm{c}^{2}-\dot{\mathrm{r}}_{2}^{2} /\left(2 \mathrm{c}^{2}\right)}{1+\Phi_{1} / \mathrm{c}^{2}-\dot{\mathrm{r}}_{1}^{2} /\left(2 \mathrm{c}^{2}\right)} \frac{\mathrm{dt}}{\mathrm{dt}}
$$

$t$ and $t^{\prime}$ being the epochs of emission and reception, respectively, in coordinate time. By

$$
t^{\prime}-t=\frac{1}{c}\left|\underline{r}_{1}(t)-\underline{r}_{2}\left(t^{\prime}\right)\right|
$$


one has

$$
\frac{d t^{\prime}}{d t}=\frac{1+\underline{k} \dot{r}_{1}(t) / c}{1+\underline{k} \dot{r}_{2}\left(t^{\prime}\right) / c}
$$

with

$$
\underline{k}=\frac{\underline{r}_{1}(t)-\underline{r}_{2}\left(t^{\prime}\right)}{\left|\underline{r}_{1}(t)-\underline{r}_{2}\left(t^{\prime}\right)\right|}
$$

The relation Eq. (62) (with Eq. (64)) is of ten used in practice. It contains two instants $t$ and $t$. Sometimes it may be useful to rewrite this relation as the function of one single argument. Denoting

$$
\underline{R}(t)=\underline{r}_{1}(t)-\underline{r}_{2}(t)
$$

one obtains

$$
t^{\prime}-t=\frac{R(t)}{c}-\frac{1}{c^{2}} \underline{R} \underline{\dot{r}}_{2}+\ldots=\frac{R\left(t^{\prime}\right)}{c}-\frac{1}{c^{2}} \underline{R} \underline{\dot{r}}+\ldots .
$$

Taking the derivative dt'/dt yields two expressions which are equivalent to Eq. (62):

$$
\begin{aligned}
& \frac{\delta \tau^{\prime}}{\delta \tau}=1+\frac{\Phi_{2}-\Phi_{1}}{c^{2}}+\frac{\dot{\mathrm{R}}(\mathrm{t})}{\mathrm{c}}+\frac{1}{2 \mathrm{c}^{2}} \underline{\dot{\mathrm{R}}}^{2}-\frac{1}{\mathrm{c}^{2}} \underline{\mathrm{R}} \ddot{\mathrm{r}}_{2}+\ldots, \\
& \frac{\delta \tau}{\delta \tau^{\prime}}=1+\frac{\Phi_{1}-\Phi_{2}}{\mathrm{c}^{2}}-\frac{\dot{\mathrm{R}}\left(\mathrm{t}^{\prime}\right)}{\mathrm{c}}+\frac{1}{2 \mathrm{c}^{2}} \underline{\mathrm{R}}^{2}+\frac{1}{\mathrm{c}^{2}} \stackrel{\ddot{\mathrm{R}}}{\underline{\mathrm{r}}_{1}}+\ldots .
\end{aligned}
$$

In the second order terms, the difference between $t$ and $t^{\prime}$ is, of course, insignificant.

In the integrated Doppler effect (Boucher 1978) the measurable quantity is the difference between the number of, impulses generated at point 2 with the frequency $f_{2}$ during the proper time interval $\left(\tau_{1}, \tau_{2}\right)$ and the number of impulses emitted at point 1 with the frequency $f_{1}$ and received at point 2 with the frequency $f_{12}$. Mathematically, this quantity equals

$$
\mathrm{N}=\int_{\tau_{1}^{\prime}}^{\tau !}\left(\mathrm{f}_{2}-\mathrm{f}_{12}\right) \mathrm{d} \tau^{\prime}
$$


Evidently,

$\int_{\tau^{\prime} 1}^{\tau_{1}^{\prime} 2} \mathrm{f}_{12} \mathrm{~d} \tau^{\prime}=\int_{\tau_{1}}^{\tau_{1}^{2}} \mathrm{f}_{1} \mathrm{d \tau}=\mathrm{f}_{1} \int_{\tau_{1}}^{\tau} \mathrm{d}$

and

$\int_{\tau_{1}}^{\tau} d \tau=\int_{\tau_{1}^{\prime}}^{\tau_{j}^{\prime}} \frac{d \tau}{d \tau^{\prime}} d \tau^{\prime}$.

Considering Eq. (69), the final result is

$$
\begin{aligned}
& N=\left(f_{2}-f_{1}\right)\left(\tau^{\prime}{ }_{2}-\tau^{\prime}{ }_{1}\right)+\frac{1}{c} f_{1}\left[R\left(\tau{ }_{2}^{\prime}\right)-R\left(\tau{ }^{\prime}{ }_{1}\right)\right]- \\
& -\frac{1}{\mathrm{c}^{2}} \mathrm{f}_{1} \int_{\tau^{\prime}}^{\tau_{1}^{\prime}}\left(\Phi_{1}-\Phi_{2}+\frac{1}{2} \dot{\mathrm{R}}^{2}+\underline{\mathrm{R}} \ddot{\mathrm{r}}_{1}\right) \mathrm{d} \tau^{\prime} \text {. }
\end{aligned}
$$

Ashby and Allan (1979) have shown that one may use the coordinate time of the geocentric reference frame in the problem of clock synchronization in terrestrial space. This is of advantage in navigation, and with the aid of navigation satellite systems like NAVSTAR as well. Consider three moving objects, a navigation satellite 1 , a user 2 , and a ground station 3. The clocks of all three objects indicate individual proper times $t, t^{\prime}, t^{\prime \prime}$, respectively. These may be converted to coordinate time for each object by a formula such as the following

$$
\Delta t=\int\left(1-\frac{1}{c^{2}} \Phi+\frac{1}{2 c^{2}} \dot{r}^{2}\right) d \tau
$$

in conjunction with the transformations of the integral function for the satellite and the ground station, cf. Ashby and Allan (1979).

At the epoch $t_{0}$ of epprdinate time let the clocks $1,2,3$ be synchronized. This means that the readings $\tau_{1} \tau_{1}, \tau_{1}$, respectively, in their individual proper times conform to the epoch $t_{0}$ of coordinate time. The satellite 1 transmits signals periodic in its proper time $\tau$. Consider a signal emitted by the satellite at the moment $\tau_{2}$ in its own time-scale $\tau$ and received by the user at the moment $\tau_{2}$ in the user's time-scale $\tau^{\prime}$. The corresponding epochs of coordinate time are $t_{1}$ and $t_{2}$, respectively. Then

$$
t_{1}-t_{0}=\tau_{2}-\tau_{1}+\frac{1}{c^{2}} \int_{\tau_{1}}^{\tau_{2}}\left(\frac{1}{2} \ddot{r}_{1}^{2}-\Phi_{1}\right) d \tau,
$$




$$
\mathrm{t}_{2}-\mathrm{t}_{0}=\tau_{2}^{\prime}-\tau_{1}^{\prime}+\frac{1}{\mathrm{c}^{2}} \int_{\tau_{1}^{\prime}}^{\tau_{2}^{\prime}}\left(\frac{1}{2} \dot{\mathrm{r}}_{2}^{2}-\Phi_{2}\right) \mathrm{d} \tau^{\prime} \text {. }
$$

According to Eq. (67)

$$
\mathrm{t}_{2}-\mathrm{t}_{1}=\frac{1}{\mathrm{c}} \mathrm{R}_{12}\left(\mathrm{t}_{2}\right)-\frac{1}{\mathrm{c}^{2}} \underline{\mathrm{R}}_{12} \dot{\mathrm{r}}_{1}+\ldots, \underline{\mathrm{R}}_{12}(\mathrm{t})=\underline{\mathrm{r}}_{1}(\mathrm{t})-\underline{\mathrm{r}}_{2}(\mathrm{t}) \text {. }
$$

From Eqs. (74) and (75) follows

$$
\begin{aligned}
\mathrm{R}_{12}\left(\mathrm{t}_{2}\right) & =\mathrm{c}\left(\tau_{2}^{\prime}-\tau_{1}^{\prime}\right)-\mathrm{c}\left(\tau_{2}-\tau_{1}\right)+\frac{1}{2} \int_{\tau_{1}^{\prime}}^{\tau_{2}^{\prime}}\left(\frac{1}{2} \dot{\mathrm{r}}_{2}^{2}-\Phi_{2}\right) \mathrm{d} \tau^{\prime}- \\
& -\frac{1}{\mathrm{c}} \int_{\tau_{1}}^{\tau_{2}}\left(\frac{1}{2} \dot{\mathrm{r}}_{1}^{2}-\Phi\right) \mathrm{d} \tau+\frac{1}{\mathrm{c}} \underline{\mathrm{R}}_{12} \dot{\mathrm{r}}_{1} .
\end{aligned}
$$

The epochs $\tau_{1}, \tau_{2}, \tau_{1}^{\prime}, \tau_{2}^{\prime}$ are known quantities and the integral as well as the last term in Eq. (77) may be calculated with their known approximate values. In combination with three analogous relations for three other satellites, this enables us to determine the accurate coordinates of the user and the time correction to his clock (Spilker 1978). Similar expression may be derived for the relationship between the satellite 1 and the ground station 3.

Finally, in radiointerferometry on the surface of the Earth the dependence Eq. (56), by Brumberg's (1981b) method leads to the following expression for the coordinate time delay betwen the epochs $t_{1}$ and $t_{2}$ respectively, of the reception of a signal by two terrestrial stations

$$
\begin{aligned}
c\left(t_{1}-t_{2}\right)= & \frac{\underline{s} \underline{d}}{1+\frac{1}{c} \underline{s}\left[\underline{r}_{1}\left(t_{1}\right)+\dot{\rho}_{2}\left(t_{2}\right)\right]}+m_{1}\left[\frac{\gamma+1}{\rho_{1}+\underline{s}(\underline{R}+\underline{\rho})}-\frac{\alpha}{\rho_{1}}\right](\underline{s} \underline{d})+ \\
& +\frac{m_{2}}{R}\left[(\gamma+1) \frac{(\underline{R}+R \underline{s}) \underline{d}}{R+\underline{R} \underline{s}}-\alpha(\underline{s} \underline{d})+\frac{\alpha}{R}(\underline{R} \underline{s})(\underline{R} \underline{d})\right] .
\end{aligned}
$$

Here, $\underline{r}_{1}$ is the position vector of the center of mass of the Earth with respect to the barycenter Sun-Earth, $\underline{R}$ is the heliocentric position vector of the center of mass of the Earth, $\underline{\rho}_{i}$ is the geocentric position vector of the station $i, d=\underline{\rho}_{2}\left(t_{1}\right)-\underline{\rho}_{1}\left(t_{1}\right)$ : is the baseline vector, $\underline{s}=\underline{r}_{0} / r_{0}$ is the direction to the radio source from the barycenter Sun-Earth. In harmonic coordinates, by virtue of (6) 


$$
\underline{\tilde{d}}=\underline{\mathrm{d}}-\alpha\left(\frac{\mathrm{m}_{1}}{\rho_{1}}+\frac{\mathrm{m}_{2}}{\mathrm{R}}\right) \underline{\mathrm{d}}+\alpha \frac{\mathrm{m}_{2}}{\mathrm{R}^{3}}(\underline{\mathrm{R}} \underline{\mathrm{d}}) \underline{\mathrm{R}}
$$

demonstrating that Eq. (78) is coordinate independent.

\section{Conclusion}

One may easily obtain numerical estimates of the above described reductions made on the basis of the values: $m_{2}=1.5 \mathrm{~km}, m_{1}=0.5 \mathrm{~cm}$ (gravitational radii of the Sun and the Earth, respectively). At the distance of the radius of the terrestrial orbit $R$ and radius of the Earth $\rho$ one has $m_{2} / R=10^{-8}, m_{1} / \rho=10^{-9}$. These values characterize the order of the relativistic effects in comparison with the main Newtonian terms.

The most timely questions of relativistic astrometry seem to be the relativistic aspects of the astronomical reference systems and the relativistic effects in the rotational motion of the Earth. These questions have been dealt with in many investigations but much remains to be done to find these solutions best suited to astronomical practice. 


\section{Appendix: Influence of the Moon and the Sun}

Let us show that in geodynamical problems of clock synchronization and navigational measurements, the influence of the Moon and the Sun may be neglected to an accuracy of $10^{-14}$ in frequency. This has been demonstrated by Ashby and Allan (1979) but in a rather complicated way considering several effects which mutually cancel each other. The following technique, which has to do with the relationship between the Earth's proper time and coordinate barycentric time (Moyer 1981), leads directly to the same conclusion.

In the barycentric system with respect to the Sun, the Earth and the Moon Eq. (1) yields, sufficiently accurate for the problems under consideration

$$
g_{o o}=1-\frac{m_{E}}{\Delta_{E}}-\frac{m_{S}}{\Delta_{S}}-\frac{m_{L}}{\Delta_{L}}, g_{o i}=0, g_{i j}=-\delta_{i j}
$$

$\Delta_{E}, \Delta_{S}, \Delta_{L}$ being the distances to Earth (E), Sun (S) and Moon (L) respectively. The proper time $t$ of clock A situated on the surface of the Earth or on the satellite will be

$$
\mathrm{d} \tau=\left[1-\frac{\mathrm{m}_{\mathrm{E}}}{\mathrm{r}_{\mathrm{A}}^{\mathrm{E}}}-\frac{\mathrm{m}_{\mathrm{S}}}{\mathrm{r}_{\mathrm{A}}^{\mathrm{s}}}-\frac{\mathrm{m}_{\mathrm{L}}}{\mathrm{r}_{\mathrm{A}}^{\mathrm{L}}}-\frac{1}{2 \mathrm{c}^{2}}\left(\dot{\mathrm{r}}_{\mathrm{A}}^{\mathrm{c}}\right)^{2}\right] \mathrm{d} \tau \text {. }
$$

Here, $\mathrm{r}_{\mathrm{I}}^{\mathrm{J}}$ denotes the position vector of the body I with respect to the body J. Index $\mathrm{C}$ refers to the Sun-Earth-Moon barycenter.

$r_{A}^{S}$ and $r_{A}^{L}$ are expanded in powers of $r_{A}^{E} / r_{E}^{S}$ and $r_{A}^{E} / r_{L}^{E}$, respectively, taking into account that

$$
\underline{r}_{A}^{S}=\underline{r}_{A}^{E}+\underline{r}_{E}^{S}, \quad \underline{r}_{A}^{L}=\underline{r}_{A}^{E}-\underline{r}_{L}^{E}
$$

On the other hand

$$
\dot{\mathbf{r}}_{\mathbf{A}}^{\mathbf{C}}=\dot{\vec{r}}_{\mathrm{A}}^{\mathrm{E}}+\dot{\mathbf{r}}_{\mathrm{E}}^{\mathrm{C}}
$$

and the term with the mixed velocities is transformed as follows

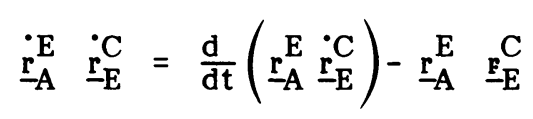

where, by virtue of the Newtonian equations

$$
\ddot{r}_{E}^{C}=-\frac{M_{S}}{\left(r_{E}^{S}\right)^{3}} \underline{r}_{E}^{S}+\frac{M_{L}}{\left(r_{L}^{E}\right)^{3}} \underline{r}_{L}^{E} \text {. }
$$


The mixed terms proportional to

$$
\begin{aligned}
& \mathrm{d} \tau=\left[1-\frac{\mathrm{m}_{\mathrm{E}}}{\mathrm{r}_{\mathrm{A}}^{\mathrm{E}}}-\frac{\mathrm{m}_{\mathrm{S}}}{\mathrm{r}_{\mathrm{E}}^{\mathrm{S}}}-\frac{\mathrm{m}_{\mathrm{L}}}{\mathrm{r}_{\mathrm{L}}^{\mathrm{E}}}-\frac{1}{2 \mathrm{c}^{2}}\left(\frac{\dot{\mathrm{r}}_{\mathrm{A}}^{\mathrm{E}}}{{ }^{2}}\right)^{2}-\frac{1}{2 \mathrm{c}^{2}}\left(\dot{\mathrm{r}}_{\mathrm{E}}^{\mathrm{C}}\right)^{2}\right. \\
& \left.-\frac{1}{\mathrm{c}^{2}} \frac{\mathrm{d}}{\mathrm{dt}}\left(\begin{array}{cc}
\underline{r}_{\mathrm{A}}^{\mathrm{E}} & \dot{\bullet}_{\mathrm{E}}^{\mathrm{C}}
\end{array}\right)\right] \mathrm{dt} \text {. }
\end{aligned}
$$

A similar relation between $\mathrm{d \tau}^{\prime}$ and $\mathrm{dt}^{\prime}$ may be established for the second clock $\mathrm{A}^{\prime}$. If $t$ is the moment of emission of the signal at point $A$, and $t^{\prime}$ is the moment of its reception at point $A^{\prime}$ then

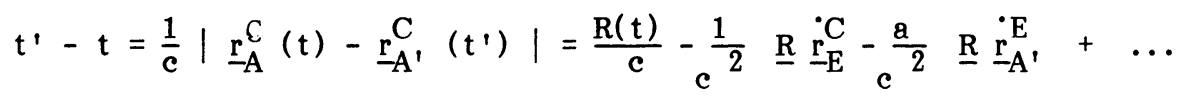

with

$$
\underline{R}(t)=\underline{r}_{A}^{E}(t)-\underline{r}_{A^{\prime}}^{E}(t)
$$

Hence,

$$
\frac{\mathrm{dt}^{\prime}}{\mathrm{dt}}=1+\frac{\dot{\mathrm{R}}(\mathrm{t})}{\mathrm{c}}-\frac{1}{\mathrm{c}^{2}} \frac{\mathrm{d}}{\mathrm{dt}}\left(\underline{\mathrm{R}} \dot{\mathrm{r}}_{\mathrm{E}}^{\mathrm{C}}\right)-\frac{1}{\mathrm{c}^{2}} \dot{\mathrm{r}}_{\mathrm{A}}^{\mathrm{E}} \dot{\mathrm{r}}_{\mathrm{A}^{\prime}}^{\mathrm{E}}+\frac{1}{\mathrm{c}^{2}}\left(\dot{\mathrm{r}}_{\mathrm{A}^{\prime}}^{\mathrm{E}}\right)^{2}-\frac{1}{\mathrm{c}^{2}} \underline{\mathrm{R}}_{\mathrm{r}_{\mathrm{A}^{\prime}}}^{\mathrm{E}} .
$$

Therefore,

$$
\frac{\mathrm{d} \tau^{\prime}}{\mathrm{d} \tau}=1-\frac{\mathrm{m}_{\mathrm{E}}}{\mathrm{r}_{\mathrm{A}^{\prime}}^{\mathrm{E}}}+\frac{\mathrm{m}_{\mathrm{E}}}{\mathrm{r}_{\mathrm{A}}^{\mathrm{E}}}+\frac{\dot{\mathrm{R}}(\mathrm{t})}{\mathrm{c}}+\frac{1}{2 \mathrm{c}^{2}} \underline{\mathrm{R}}^{2}-\frac{1}{\mathrm{c}^{2}} \underline{\mathrm{R}}_{-} \ddot{\mathrm{r}}_{\mathrm{A}^{\prime}}^{\mathrm{E}} .
$$

This expression contains only the geocentric quantities and coincides with Eq. (68). (In this approximation there is no difference in coordinate time for geocentric and barycentric systems.)

The neglected terms

$$
\frac{m_{S}}{\left(r_{E}^{S}\right)^{3}}\left(r_{A}^{E}\right)^{2}, \frac{m_{L}}{\left(r_{L}^{E}\right)^{3}} \quad\left(r_{A}^{E}\right)^{2}
$$

are of the order $10^{-24}\left(\mathrm{r}_{\mathrm{A}}^{\mathrm{E}}\right)^{2}$ (for the Sun with the coefficient $1 / 2, \mathrm{r}_{A}^{\mathrm{E}}$ in units of $\mathrm{km}$ ). For satellites with a geocentric distance $\mathrm{r}_{\mathrm{A}}^{\mathrm{E}}=3.10^{4} \mathrm{~km}$, this is of the order $10^{-15}$. 


\section{Acknowledgement}

The author is extremely indebted to Professor Heinrich K. Eichhorn for editing the English version of the manuscript.

\section{References}

Aoki S., Fujimoto M.K. and Fukushima T. 1982. An Operational Procedure of the International Atomic Time. Meeting Commission 31, IAU Gen. Assembly.

Arifov L.Ya. 1983. General Theory of Relativity and Gravitation. Tashkent (in Russian).

Arifov L.Ya. and Kadyev R.K. 1968. Astron. Zh. (USSR) 45, 1114.

Ashby N. and Allan D.W. 1979. Radio Science 14, 649.

Ashby N. and Bertotti B. 1983. 10th Int. Conf. Gen. Relat. and Gravit., Contrib. Pap. 2, 948.

Ashby N. and Bertotti B. 1984. Phys. Rev. Lett. 52, 485.

Boucher C. 1978. Relativistic Correction to Satellite Doppler Observation. Meeting IAGSSG 4:45.

Brumberg V.A. 1981a. In: Reference Coordinate Systems for Earth Dynamics (E.M. Gaposchkin and B. Kolaczek eds.), 238, Reidel.

Brumberg V.A. 1981b. Astron. Zh. (USSR) 58, 181.

Brumberg V.A. and Ivanova T.V. 1982. In: Sun and Planetary System (W. Fricke and G. Teleki eds.), 423, Reidel.

Fujimoto M.K., Aoki S., Nakajima K., Fukushima T. and Matsuzaka S. 1982. Proc. Symp. No. 5 IAG, 26.

Ivanitskaja O.S. 1979. Lorentzian Basis and Gravitational Effects in the Einsteinian Theory of Gravitation. Minsk (in Russian).

Kostyukovich N.N. 1982. Vestnik AN BSSR, phys.-mat. ser., No. 5, 84.

Krasinsky G.R., Pit'eva E.V., Sveshnikov M.L. and Sveshnikova E.S. 1981. Doklady AN USSR 261, 1320.

Lestrade J.F. and Bretagnon P. 1982. Astron. Astrophys. 105, 42.

Lestrade J.F. and Chapront-Touze' M. 1982. Astron. Astrophys. 116, 75.

Manasse F.K. and Misner C.W. 1963. J. Math. Phys. 4, 735.

Mast C.B. and Strathdee J. 1959. Proc. R. Soc. A252, 476. 
Mikhailov A.. 1969. Astron. Zh. (USSR) 46, 454.

Mbller C. 1972. The Theory of Relativity. Clarendon Press, Oxford.

Moyer T.D. 1981. Cel. Mech. 23, 33, 57.

Murray C.A. 1981. Mon. Not. R.A.S. 195, 639.

Oesterwinter C. and Cohen C.J. 1972. Cel. Mech. 5, 317.

Pavlov N.V. 1984. Astron. Zh. (USSR) 61, 385, 600.

Spilker J.I. 1978. Navigation 25, 121.

Synge J.L. 1960. Relativity: The General Theory. North-Holland.

Vladimirov Yu. S. 1982. Systems of Reference in Gravitation Theory. Moscow (in Russian).

Vyblyj Yu. P. and Kostyukovich N.N. 1982. Appl. Math. Mech. (USSR) 46, 621.

Will C.M. 1981. Theory and Experiment in Gravitational Physics. Cambridge Univ. Press. 